\title{
Carnets
}

Revue électronique d'études françaises de l'APEF

Première Série - 2 Numéro Spécial 10-11 | 2011

D'un Nobel l'autre

\section{L'étrangeté, l'exil et l'amour chez Beckett}

\section{Béryl Schlossman}

\section{(2) OpenEdition}

Journals

Édition électronique

URL : http://journals.openedition.org/carnets/5582

DOI : 10.4000/carnets.5582

ISSN : 1646-7698

Éditeur

APEF

Édition imprimée

Date de publication : 1 janvier 2011

Pagination : 123-132

Référence électronique

Béryl Schlossman, «L'étrangeté, l'exil et l'amour chez Beckett », Carnets [En ligne], Première Série - 2 Numéro Spécial 10-11 | 2011, mis en ligne le 16 juin 2018, consulté le 19 avril 2019. URL : http:// journals.openedition.org/carnets/5582 ; DOI : 10.4000/carnets.5582

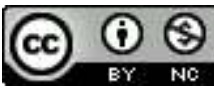

Carnets est mis à disposition selon les termes de la licence Creative Commons - Atribution - Pas d'utilisation commerciale 4.0 International. 


\title{
L'ETRANGETE, L'EXIL ET L'AMOUR CHEZ BECKETT
}

BERYL SCHLOSSMAN

Carnegie Mellon University

bfs@andrew.cmu.edu

\begin{abstract}
Résumé
L'oeuvre de Samuel Beckett, c'est un éventail de styles littéraires et de sujets qui résonnent encore parmi les plus actuels. Beckett est porteur d'une modernité engagée, post-moderne pour certains mais surtout ancrée dans l'écriture comme lieu d'une résistance à toute habitude de complaisance subjective. La lecture de Premier amour (1945) montre comment Beckett nous entraine encore, toujours, dans l'étrangeté de l'être et dans la dimension poétique des traversées de langues. Ses jeux de l'autobiographie fictive, depuis le foisonnement et le labyrinthe des premiers romans jusqu'au minimalisme de ses dernières années, marquent l'histoire unique d'un prix Nobel qui remonte à 1969.
\end{abstract}

\begin{abstract}
The styles and subjects of Samuel Beckett's works continue to resonate in contemporary literature and theater: Beckett's modernity is contemporary with current 'post'-modern sensibility. Beckett's radical engagement with modernity is anchored in writing as the site of resisting any habits of subjective selfpity or complacency. A reading of First Love (first written in French, 1945) illustrates the ways that Beckett moves us beyond the predictable, into the strangeness of being and the poetic dimensions of language. From the rich play of language in the labyrinth of his early novels through the minimalism of his later years, fictional autobiography marks the unique history of his Nobel Prize awarded in 1969.
\end{abstract}

Mots-clés: Samuel Beckett, modernisme, fiction, la nouvelle, poétique, l'exil, l'amour Keywords: Samuel Beckett, modernism, fiction, short story, poetics, exile, love 
Les romans, le théâtre, les poèmes et d'autres écrits signés par Samuel Beckett présentent un éventail de styles littéraires et de sujets qui résonnent encore parmi les plus actuels. Le modernisme dont Beckett a hérité, en quelque sorte, et où il a reconnu la nouveauté de la littérature moderne depuis Flaubert et d'autres, l'a provoqué à trouver sa voix propre puis à la transformer en une écriture de l'inattendu et de l'insolite. Son oeuvre en plusieurs genres est construite d'un ensemble de voix et d'appels aux non-voix (aux voix tues) du silence. Ce sont les instances - très précises même quand elles ne proposent du sens que la pente vers l'opacité, le vague, et l'énigmatique - de ce qui se parle et s'écrit chez Beckett, dans des monologues simples ou pluriels, et parfois en dialogues. Son écriture est d'un aspect singulier au même titre que l'écriture de Kafka, d'Artaud, de Pessoa, de Proust, de Brecht, de Woolf, ou de Joyce, entre autres, et comme eux, il s'est engagé dans la recherche d'une modernité désormais acquise et dont l'influence contemporaine est incontestable.

Moderniste, son oeuvre est dans le sillage post-moderne par sa mise en question des traditions littéraires et des fonctions narratives auxquelles, pourtant, son écriture a l'air de tenir. Cette oeuvre habite pleinement, comme celles de Joyce ou de Proust, que Beckett parodiait avec verve et tendresse, le temps et l'espace de l'allégorie moderne dérivée du modernisme du milieu du dix-neuvième siècle. Plénitude qui ne réjouit pas plus ses personnages que ceux de Kafka.

Le présent essai sur l'étrangeté, l'exil et l'amour se propose au coeur d'un programme personnel de lecture de Beckett, aux principes suivants: 1. L'oeuvre est ancrée dans l'écriture considérée comme un acte autonome et rigoureux, d'une part; 2. D'autre part, l'écriture est ancrée dans une expérience littéraire riche de résonances et d'échos de plusieurs traditions; 3 . En même temps, l'oeuvre tend vers le minimalisme lié à un ensemble de refus qui seront à déterminer; 4. A partir de ces trois principes, l'oeuvre explore la souffrance et le rire comme voies d'accès à l'étrangeté de l'être; et 5 . la langue est traitée dans sa dimension poétique mais parlée, et foncièrement anti-romantique, que ce soit en français ou en anglais.

Avec le temps, l'écriture de Beckett allait de plus en plus vers une forme de minimalisme, tandis que la richesse symbolique qui se donnait dans les oeuvres des premières années devenait progressivement de moins en moins visible. Or malgré ces variations, l'oeuvre de Beckett est une: elle se présente comme champs de résistance à toute habitude de complaisance subjective. Pour aller jusqu'au bout de la vie et de la mort, de la conscience et de toute l'opacité de l'expérience humaine, Beckett nous entraine encore, toujours, dans des traversées de l'étrangeté de l'être.

L'Irlande le fait fuir et revenir, inlassablement. Anglo-irlandais au départ et pour toujours, l'oeuvre est marquée par l'exil irlandais, la violence de l'histoire du pays, et l'amour 
de I'Irlande. Parisien et francophone d'adoption, Beckett est marqué tout autant par une certaine liberté parisienne et intellectuelle, par la philosophie, par l'exil intérieur et par la guerre. Ses jeux d'autofiction et d'autobiographie fictive, depuis le foisonnement et le labyrinthe des premiers romans jusqu'au minimalisme de ses dernières années, marquent I'histoire unique d'un prix Nobel qui remonte à 1969. Si cela ne suffisait pas, il faudrait y ajouter tous les malentendus concernant l'identité littéraire de l'oeuvre et le statut uniquement paradoxal de l'auteur voyageur, francophone mais d'origine anglophone, dublinois et irlandais - ni français ni anglais, en somme - pour regarder attentivement en quoi sans Beckett, il n'y aurait peut-être pas eu les suites parisiennes et autres du Nouveau Roman, dont il était censé être un représentant, lui qui ne représentait rien ni personne à quelque titre officiel que ce soit. Et cela, si on peut le dire ainsi, à coeur joie, puisque ce refus de porter des étiquettes ou de soutenir littérairement des parti-pris, c'était presque une signature chez lui, Samuel Beckett. Ce qui ne changeait rien, d'ailleurs, à ses engagements. La publicité ne l'attirait aucunement. L'auteur a reçu — sans l'avoir agréé — son prix Nobel comme une vraie catastrophe. A cette occasion, il n'a pas fait signe au pays d'origine, c'est un symptôme révélateur, ni au pays adoptif non plus. L'idéologie, peut-être, ou bien l'Histoire (avec majuscule) invivable se serait transformée, pour ce témoin singulier de la deuxième guerre mondiale, en consommation idéologique, et que Beckett n'appréciait pas, lui qui (depuis longtemps) s'était élaboré un art de la résistance dont les échos se prolongent jusqu'à nous.

L'art de Beckett est d'une certaine façon bien plus français (intellectuellement) que son auteur, qui prolonge une tradition qui remonte à Racine et Sévigné autant qu'à Descartes. C'est un point en commun avec Proust, d'ailleurs, pour qui l'identité est à refaire dans l'écriture. La notion de "littérature-monde en français" est loin du monde de Beckett même si lui va dans ce sens. II est bien plus proche du roman de l'inconscient qui, chez Proust, donne lieu à la tentative de refaire la société en édifice du Temps, rendu visible, construit d'après les plans du désir et défait par ces mêmes plans. Chez Beckett, le temps balaie les structures identitaires, il ronge tout.

Dans la perspective d'une "littérature-monde en français" — ou bien partiellement en français, car l'identité (linguistique, entre autres) est une question plutôt qu'une réponse l'oeuvre de Samuel Beckett nous engage à regarder de près son actualité littéraire. De façon singulière, sinon unique, Beckett habite deux langues principales un peu comme un passeur qui va, sans se poser des questions d'origine identitaire ni d'appartenance communautaire, sans cesse d'une rive à l'autre en transportant il ne sait pas trop quels clients, quelles marchandises, quelles nouvelles. En cela, l'influence de l'oeuvre de James Joyce est sans doute exemplaire. Joyce considérait les langues un peu comme une montagne qu'il creusait de tous les côtés pour se retrouver dans un passage souterrain leur permettant de 
communiquer secrètement entr'elles. La subversion n'est pas la même, mais la fréquentation de l'oeuvre de Joyce (Les Dublinois, les poèmes et les essais critiques, Le Portrait de l'artiste en jeune homme, Ulysse et Finnegans Wake) ainsi que de leur auteur, tout aussi passionnément dublinois et irlandais que Beckett lui-même, est plus que suggestive.

Grâce à Marcel Proust et James Joyce, ses deux antécédents modernistes les plus incontournables, certains jeux de l'autobiographie et de l'autofiction viennent dans la francophonie depuis la plume d'un auteur qui n'était pas tellement francophone, au départ de sa vie à Paris, où bien des apatrides arborant des accents les moins passe-partout du monde vivaient l'exil et, enfin, l'après-guerre. Entre la fièvre enthousiaste et l'ironie grinçante de l'Histoire, ce cauchemar d'où l'on n'arrive pas à se réveiller, le choix de Beckett, c'est de prendre la sortie marquée catastrophe. On se sauve mais le cauchemar de l'histoire continue, donc on ne sera pas sauvé - sauf si l'on arrive `se faufiler, à jouer, à faire le pitre. Chute dans le temps, scansion poétique, voilà la voie d'après Beckett, à dire vrai, remplie d'exploits mais sans fictions de gloire. Le temps de cette catastrophe se remplit surtout d'attentes - longues, ennuyeuses, foudroyantes, mystérieuses, emprisonnantes, pirates, mortelles, et, de façon surprenante, amoureuses. Mais l'amour, s'il est très souvent évoqué par Beckett, n'est pas souvent consacré par la mise en scène théâtrale. Le plus souvent, l'amour est enfoui dans un passé opaque et trouble, d'où il se manifeste de façon bouleversante et surtout invisible. Les attentes amoureuses ne durent pas suffisament longtemps pour s'inscrire dans le moment actuel. L'amour chez Beckett est consacré par le passé et parfois par la dimension romanesque du souvenir. L'attente amoureuse fait rarement partie du temps présent, ce serait plutôt une catastrophe en plus, une sorte d'antiquité que l'on va oublier, qui est déjà à moitié oubliée.

Chez Beckett, le temps perdu n'est jamais retrouvé de façon proustienne, avec les promesses de l'Art et les fruits subtils de l'Amour, ni de façon joycienne, où il y a en fin de compte une résurrection bien sonnante, avec extase et joies de toutes sortes. Le modèle de Pâques et de ses passages par la Nuit arrive tout de même à un Jour saisissant, chez Proust autant que chez Joyce, quoique différemment, suite au vocabulaire esthétique et théologique du modernisme. Le catholicisme y est pour quelque chose chez Proust autant que chez Joyce. Malgré ses lectures, depuis Dante jusqu'aux modernistes, Beckett est plus marqué par le Protestantisme irlandais. II ne se voit pas plus sauter de joie dans l'écriture que dans les grandes émotions de la vie, d'où des centaines d'anecdotes célèbres. Son oeuvre, ses personnages, habitent un monde (des mondes) où l'enthousiasme serait mal placé. Même le ton apocalyptique chez Beckette semble dangereusement vivace. Beckett habite d'autres mondes que l'enthousiasme.

Dans le monde de Beckett, il s'agit de souffrance et de cruauté, de tendresse, d'abjection, d'obscénité et de faiblesse narcissique, de lectures et d'échos discrets, des 
couleurs mais surtout du noir et du gris. Par contre il y a l'humour, la comédie, le vaudeville, les lazzi, et par moments, une sorte de victoire bien étrange. Le support humain des émotions et des rares événements - des pensées - est peu de chose, une loque humaine, dont on s'occupe ou que l'on laisse tomber. L'exil domine les mondes de Beckett, la prison virtuelle, la distance infranchissable de ce que l'on désire ou désirerait si on pouvait, seulement, désirer quelque chose. On est parti très loin mais on revient toujours, comme les étoiles, à la même place, au même exil. C'est dans ce contexte que Beckett ou ses personnages évoquent le Purgatoire.

Tout de même, il est parfois question de l'amour, qui est affecté, chez Beckett, par les conditions de l'exil de façon explicite. II n'y a pas de célébrations publiques consacrées à Eros ni d'offrandes immolées devant Psyché. Ce serait plutôt une bonne occasion de faire le deuil de soi-même, perdu non dans les souvenirs poétiques et les langueurs sensuelles de saudade, délicieuse musique qui nous prend à la gorge, mais à l'occasion, dictée par Beckett, perdu dans la chute libre de soi, dans le délire éploré du deuil du père, bref une catastrophe dont l'amour ne fait que reproduire des échos, des vagues.

L'oeuvre de Beckett part et repart, il y a tant de moments liminaires, où l'écriture semble quitter le chemin des antécédents pour nous plonger dans une nouveauté insolite tout en rappelant des éléments déjà vus. Premier amour, rédigé en français en 1945 et publié seulement en 1970, se lit comme un des points de départ de l'oeuvre beckettienne. Sa lecture nous permet d'explorer comment l'étrangeté et l'exil habitent l'oeuvre depuis ses débuts, malgré les apparences. C'est une nouvelle en prose mais fortement poétique, un drame virtuel, intérieur, qui évoque l'amour là où les dimensions du temps et de l'espace, ainsi que d'autres balises de l'identité, sont radicalement remises en question.

Le Narrateur et personnage principal de Premier Amour donne son avis: "Ce qu'on appelle l'amour c'est l'exil, avec de temps en temps une carte postale du pays, voilà mon sentiment ce soir" (Beckett, 1970: 22). Puis lorsqu'il se rend compte qu'il est amoureux de Lulu, dans une évocation de "l'affreux nom de l'amour", le Narrateur évoque la volonté de suicide tout comme il évoquait, au début du texte, le rapport entre son mariage et la mort de son père (Beckett, 1970: 26). A la mort du père, ce personnage étrange du Narrateur, mis à la porte de la maison familiale, fut désormais sans abri.

Le Narrateur commente l'amour dans le contexte de I'Irlande, de I'histoire, d'une écriture un peu spéciale de mots d'amour: il y glisse une référence aux préservatifs introuvables dans son pays natal et évoque l'amour des traces de l'histoire chez ses compatriotes (Beckett, 1970: 27). II voit les irlandais sans cesse partis en quête des traces historiques ou bien scatologiques de l'Irlande. L'amour se complique - la progéniture est inévitable mais le pays est "le paradis des sans-logis" (Beckett, 1970: 27). Paradis bien ennuyeux, tout proche d'un purgatoire sinon d'un enfer, mais ce "paradis" chez Beckett n'est 
qu'une hyperbole comique, ironique, sarcastique. On est dispensé d'extase. Si paradis il y a, on n'aura qu'à recommencer les parcours avec Watt, Vladimir, Estragon, et bien d'autres sans nom que Beckett reconnaît à l'errance, à la mélancholie, à un air de pays qui ne trompe pas - et sans doute, à la musique parlée des tons de Dublin.

L'amour - dit le premier - se complique et complique l'existence, la progéniture est inévitable, mais le Narrateur est sauvé, il se sauve dans le pays. L'Irlande, "le paradis des sans-logis", le reprend, il abandonne la femme en couches dont les cris le poursuivent à tout jamais, bien que de moins en moins clairs, il se repossède après "l'amour" pour rechercher les stations de sa croix antérieures. L'exil devient familier, un chez-soi en quelque sorte. Ce serait une parabole - une fable ou une allégorie - du monde entier, non seulement de l'Irlande.

Dans ce texte-clef de l'oeuvre, Beckett propose l'amour comme la catastrophe du moment. La solitude du personnage qui parle d'une voix narrative très particulière - un monologue, parfois interrompu par des bribes de conversation rapportée - est située entre la mort de son père et une drôle de vie de couple, une sorte de mariage, d'après lui, qui dure un temps. Ce ménage, qui tient du hasard et de l'intérêt, de l'emprisonnement et du dévouement, ne retient pas pour le sujet ou le Narrateur l'intérêt passionné qu'il manifeste au début pour le souvenir de son père, loin de là. Ce qui concerne la mort du père se situe dans un cadre presque sublime. Le récit est pourtant comique, à tout moment. Le sublime n'effleure pas la rencontre du personnage avec une femme qui bizarrement s'intéresse à lui: le sublime reste au loin, tandis que la vie en couple n'arrête pas de poser des problèmes. La trace de l'amour pourtant se révèle dans la discussion du nom propre de la femme.

Encore une fois, comme c'est le cas chez un personnage de Murphy et dans d'autres écrits, la femme qui s'intéresse à ce monsieur est une prostituée. Dans la nouvelle, elle ne tardera pas à lui prodiguer des soins quasi-maternels jusqu'au jour où elle donne le jour à un enfant, censé être de lui. Le Narrateur est clair à ce sujet, le père de l'enfant sera un client ou un autre, mais pas lui. La paternité ne retient pas son attention. II ne saurait avoir qu'un seul père, dans le monde du Narrateur, et c'est le sien.

La mort commence la nouvelle, et la naissance la termine: la mort du père lance le Narrateur dans son premier exil, et dans l'amour — si c'est bien cela, l'amour. Par la suite, le Narrateur sera clair à ce sujet - c'est bien l'amour, même si dans l'ensemble, en ce qui concerne la vie du Narrateur, ce n'est qu'un moment d'arrêt. L'inévitable grossesse indésirable, d'après le personnage qui (comme son auteur) fait allusion au problème de la contraception en Irlande au début du siècle, lancera le Narrateur vers l'exil de nouveau, vers un départ sans but. La naissance d'un autre l'expulse lui - ou bien, lui aussi - dans le monde, dans un monde avide de repères. Cet individu est encore et sera toujours fils de son père, son amour retournera toujours vers son père, mais les cris de la femme, qu'il entendait 
en prenant la fuite, le poursuivent et le dérangent. Cela n'empêche pas l'équivalence des deux expulsions, des deux exils, qui le mettent en mouvement. Tout vagabondage chez Beckett semble prendre forme ici: le père est mort, le fils prend la route; vivre chez une femme n'est pas possible à la longue, tous les sujets masculins prennent tôt ou tard la décision de quitter leur ménage improvisé, chaotique, sans points d'ancrage. Pour un point de vue sur le sublime de l'amour de la mère et au-delà, il faut attendre Krapp dans La Dernière bande.

A parcourir l'oeuvre entière de Beckett, on constate que l'amour est souvent évoqué mais rarement présenté comme possible, vrai, ou vivable, vue la capacité limitée ou limite des personnages. La sexualité est présentée de la même façon, et l'idée de l'amour érotique semble, chez Beckett, quelque chose de totalement sans espoir en ce qui concerne l'Irlande de son époque ou bien les personnages signés de sa plume (ou tapés à la machine). La catastrophe de l'amour - l'amour est démuni ou sans espoir, l'homme se trouve face à la Vénus redoutable, et tous ces personnages, homme ou femme pour la plupart, se voient confrontés par le semblable transformé au-delà de tout image en miroir. Sans tableau. Sans une scène à jouer, et sans sortie.

L'homme confronté à l'amour à la fin de sa vie dans Malone a bien un semblable, sinon un frère dans le protagoniste de Premier amour, une sorte de vagabond plutôt jeune, qui lui-même montre le chemin aux deux bonhommes (des clowns qui ne seront jamais ni des pierrots ni des arlequins, par manque de beauté et de maîtrise?) les plus connus de Beckett, Vladimir et Estragon en attente de Godot un peu plus tard. La notion du personnage sans repères, lâché dans la nature, à la recherche non pas du temps, il est encore jeune et ne le sait pas, qu'il partira plus tard à la recherche du temps perdu, puisque pour le moment il est obligatoirement préoccupé par sa survie. Et voilà le mystère: tout de même, il s'intéresse à son semblable. Ou peut-être ce serait une dissemblable, l'autre de l'autre sexe, vu son manque d'expérience? Semblable ou pas, être humain ou avatar de Vénus la déesse si tragiquement inhumaine pour tout disciple de Racine, c'est une femme, Lulu, une étrangère comme lui, le personnage qui la rencontre. II n'arrive pas à prononcer son nom à la française, il ne peut le dire qu'à l'irlandaise ou à l'allemande peut-être, Loulou, rappel bien connu du perroquet de Flaubert.

Est-ce qu'elle incarne le Mal, vu sa profession la plus ancienne du monde? est-ce qu'on dirait qu'elle n'est qu'une fille pauvre et sans éducation, ouvrière ou 'sex-worker', tristement banale? est-ce que le sujet pas encore tout à fait vagabond en sait quelque chose, de ce qu'elle est, cette étrange semblable? Elle n'est pas tellement agréable, de toute façon, cela au moins est sûr, et lui est encore moins agréable, cela aussi est sûr. S'il y a un climat salace ou même un petit parfum de péché dans cet étrange ménage, ce n'est pas repérable par le Narrateur (vagabond et personnage principal), qui évoque son mariage au début du 
texte mais sans laisser repérer la plus petite trace, sans en donner le moindre indice. Puisque chez les personnages de Beckett on doit guetter le moindre indice, vu le drame intérieur qui occupe ses personnages d'un appétit de destruction, d'un feu incompréhensible, des besoins des plus primitives et d'une vue d'autrui qui par moments, régulièrement, frôle la psychose, même si parfois un personnage, plutôt étonné, se montre compatissant de la douleur d'autrui. Et tout cela finit bien souvent par provoquer une réaction émotive chez les spectateurs/lecteurs, et par les faire rire ou pleurer de la mise en scène de la condition dite humaine, voilà l'exploit de Beckett.

En tout cas, il finira par la fuir comme tous les autres types (ou bonhommes) de Beckett fuient à la fois Vénus, l'amour, des femmes rencontrées ou parfois aimées, comme si le refus indiquait du semblable quelqu'un ou quelque chose d'inévitablement autre, hostile, violent, menaçant, ou à la limite, impossible. II s'en va au moment même où elle - la prostituée qui l'entretient de son métier, qui lui sert des repas, qui s'occupe de lui, disait-il va mettre au monde un enfant. Ses cris dans l'enfantement lui font horreur. II prend la fuite mais il dit ne pas pouvoir les oublier, ses cris. Déjà fantôme il ne le sera que davantage. Si l'on en juge par d'autres personnages signés Beckett, il arrivera tout de même à oublier ces cris comme il oubliera celle qu'il a "aimée" de façon dérisoire, comique, enfantin, narcissique, etc. mais qui elle-même semblait si peu de chose, une putain pas tellement aimable et sans la moindre illusion sentimentale.

Elle est tout de même une sorte de semblable, qu'il appelle Anne après avoir décidé que son prénom à elle ne fera pas l'affaire. Mais du coup, qu'il oublie ou qu'il ne l'oublie pas, cette expulsion volontaire fera de lui un fantôme, un vrai, encore un. De cet état-là, chez Beckett, il n'y a pas de retour, aucun miracle, aucun espoir. L'amour n'a pas la moindre chance d'exister dans ce monde où il n'y a pas d'hommes, pas de vrais, seulement des ombres, et où s'il y a quelques femmes, un peu héroïques parfois, elles sont généralement aussi limitées que les hommes. Mais elles servent à quelque chose, elles font le service. L'exception dans tout cela - le personnage qui fait un effet, qui produit un peu de sentiment - ce serait peut-être le père.

On prend la porte, on s'expulse ou se fait expulser, et l'écran couvre tout cela d'un voile épais de fumée: on ne parle ni de désir ni d'amour, on ne parle que de naissance et de mort, d'enfants précipités vers la naissance ou des vieux sur le point de mourir. De toutes ses figures poétiques, inlassablement, Beckett mélange ou téléscope ces deux moments de la vie réduite à ses expériences les plus extrêmes, où la parole n'est pas ou plus, la parole ou le langage qui ne sauvent pas encore ou plus personne en ouvrant une possibilité de réflexion dans l'être même. Beckett jumelle ou dédouble les expériences les plus liminaires, les frontières de l'être et de la folie qu'il y a à vivre. Et à parler, puisque la parole est au centre de son enquête littéraire, de son questionnement littéraire. Les termes mêmes de 
l'existence ne mesurent pas le vide cerné par l'écriture chez Beckett. A lire la correspondance de Beckett, les voix plus intimes qu'il prend lui-même, tout-à-fait indépendants des voix qui parlent dans ses écrits, la lecture, le sport, l'amitié, la promenade, l'érotisme, la littérature, la peinture, la philosophie - l'alcool même, nectar et poison — tout ça ne change rien, ne change rien au fond.

Chez Beckett, le roman même est parti en exil, le poème devient de plus en plus court, la pièce de théâtre transportée par la violence et l'oubli donne toute son envergure à la poétique de la voix blanche, tout comme les proses, au fur et à mesure de l'oeuvre. De cette façon, on n'arrête pas de faire retour au mal/ à Malone depuis Mal vu mal dit, la dernière fleur du mal, en quelque sorte, de Beckett. Malone qui nous présente d'une façon inoubliable l'amour, la violence, Vénus la déesse de l'amour, le crayon qui fait écrire l'amoureux (brièvement) des poèmes (de MacMann) en réponse aux lettres d'amour de Moll. II est entraînée dans un enthousiasme inédit chez lui à écrire de façon poétique à son infirmière bien-aimée, mais cela ne dure pas très longtemps. Au bout d'un moment, il reprend le crayon afin de tromper la solitude de l'Homme Seul, Man Alone, ou bien à des buts littéraires que personne ne cherche à comprendre, lui le dernier. Malone ne replonge dans la poésie qu'à la fin, lorsqu'il aura mis à mort la plupart de ses personnages pour appréhender — c'est là que cela devient poétique - le moment exquis où son semblable dans la fiction prend le large et lui-même suspend le cours du temps dans une dernière petite pluie de négations, "il ne touchera jamais/ voilà jamais/ voilà voilà / plus rien" (Beckett, 1951:191).

Mais le Narrateur de Premier amour n'en est pas encore là. Pour le moment, il prend la porte, il s'enfuit afin de ne plus entendre des bruits, les cris de celle qu'il aime. II ne reviendra jamais. Ainsi est né le personnage typique de Beckett, répugnant d'étrangeté, d'exil, en voie de devenir fantôme afin de nous parler et de nous dégoûter jusqu'au bout. Le rire même va devenir plus rare, le clown qui rit et qui pleure deviendra avec les décennies une sorte de fantôme qui ne fait ni l'un ni l'autre mais qui réfléchit, et nous sert de miroir d'inquiétude, au-delà de toute identification, de toute identité, de tout delectatio morosa, même, là où la mélancholie quitte la scène devant nous pour laisser toute la place à l'étrangeté et au manque d'identité. Buster Keaton dans "Film" nous en donne un exemple saisissant, une image de la vie selon Beckett: la course aveugle entre les deux points qui chez Beckett sont des termes contaminés l'un par l'autre - la naissance est mortelle, la mort est une naissance dans le vide sans représentation. De l'enfer on peut parler inlassablement, ce serait plutôt le purgatoire d'où on n'avance pas, mais du paradis il n'y a rien à dire. 


\section{Bibliographie}

BECKETT, Samuel (1951). Malone meurt. Paris: Minuit.

BECKETT, Samuel (1965). Film. Mise en scène par Alan Schneider, cinématographie de Boris Kaufman, joué par Buster Keaton et filmé à New York en noir et blanc. 22 minutes.

BecketT, Samuel (1970). Premier amour. Paris: Minuit.

Schlossman, Beryl (1999). Objects of Desire: The Madonnas of Modernism. Ithaca et Londres: Cornell University Press.

SCHLOSSMAN, Beryl (2002). "Crossing Francophone Boundaries: Beckett's Fictions". In: TwentiethCentury Studies in Literature, vol. 26, numéro 1, pp. 101-116. 